\title{
Observation of two-photon induced photoemission optogalvanic effect
}

\author{
P R SASI KUMAR, G PADMAJA, A V RAVI KUMAR, \\ V P N NAMPOORI and C P G VALLABHAN \\ Laser Division, Department of Physics, Cochin University of Science and Technology, Cochin \\ 682 022, India
}

MS received 13 November 1990, revised 19 December 1990

\begin{abstract}
Alstract. Observation of laser induced two-photon photoemission optogalvanic (TPPOG) effect from tungsten electrode in a discharge cell using $564 \mathrm{~nm}$ radiation obtained from a pulsed dye laser is described. The magnitude of the POG signal is studied as a function of laser energy under various discharge parameters. Competition between one-photon and two-photon processes has been observed when nitrogen gas is used in the discharge cell.

Keywords Optogalvanic effect; two-photon absorption; photoemission.

PACS Na 33-80
\end{abstract}

\section{Introduction}

Optogalvanic spectroscopy (OGS) is a technique where a glow discharge is perturbed by electromagnetic radiation due to resonant energy transfer between the radiation and atoms, jons, or molecules (Green et al 1976; Smith and Schenck 1978). The energy absorbed by the species alters the plasma charge density and produces a measurable impedance change. Ambient fluctuations in discharge current usually determine the minimum detectable $O G$ signal level. $O G$ effect can also be produced by injecting electrons into the discharge via photoelectric emission from the electrode surface by laser irradiation. The photoelectrons emitted from the cathode may excite or ionize some of the atoms on its way to the anode in the discharge and produce more electrons by collisions. The effective quantum efficiency in the plasma discharge is at least $(V / d)^{1 / 2}$ ( $V$ is the applied voltage and $d$ the inter-electrode separation) times larger than that in vacuum due to the increase in the collisionally produced secondary electrons (Downey et al 1988). This photoemission optogalvanic (POG) phenomenon is a nonresonant process and since the magnitude of the signal depends on the surface composition of the electrode material, the method is useful as an in situ analytical tool for plasma electrode surface characterization using various plasma gases and different electrodes (Downey et al 1988). In this paper we report for the first time, the observation of two-photon induced photoemission optogalvanic (TPPOG) signal using pulsed dye laser.

\section{Experimental set up}

The output radiation from a pulsed dye laser (Quanta Ray PDL-2) pumped by the second harmonic of a Q-switched Nd:YAG laser (Quanta Ray DCR-11) was used as 


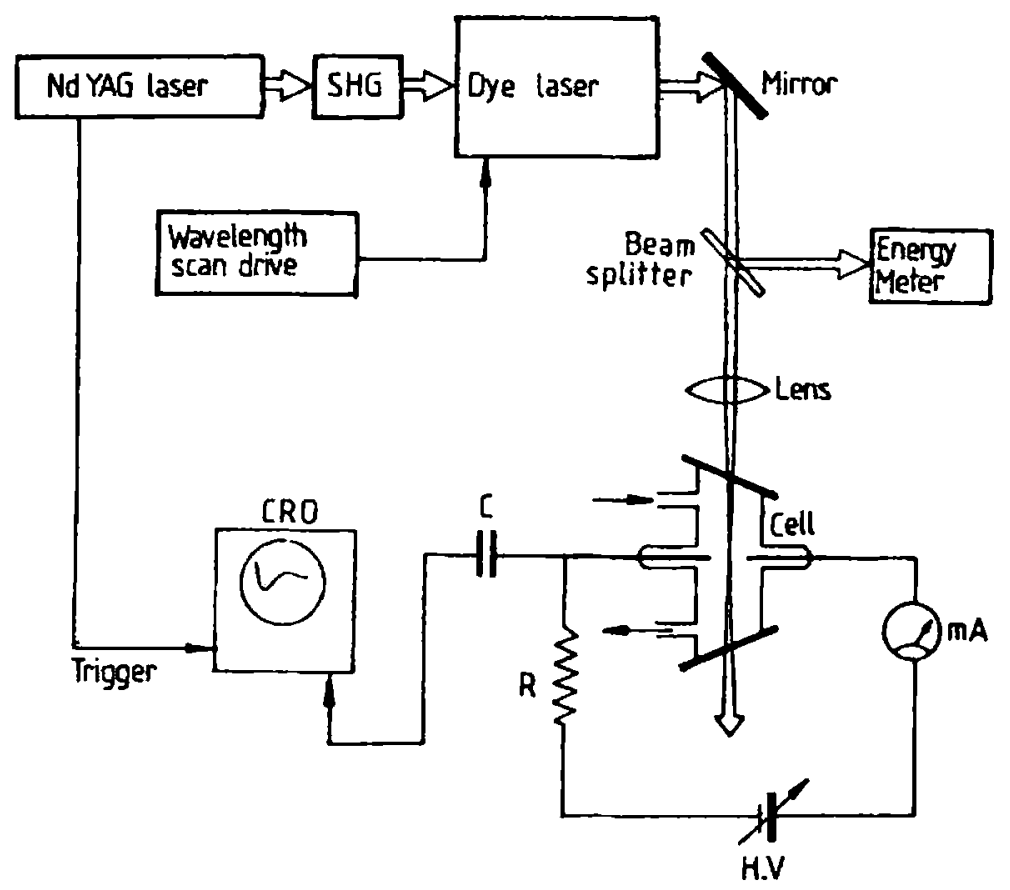

Figure l(a). Schematic diagram of the experimental set up.

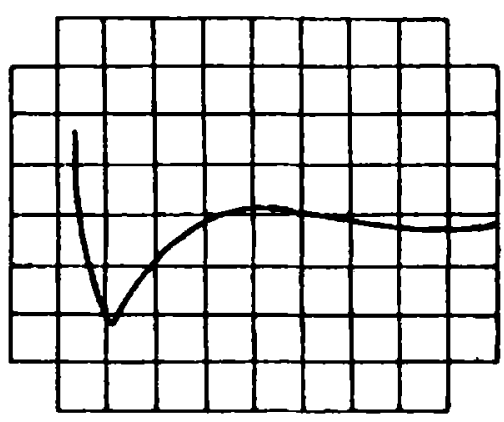

Figure 1(b). Typical CRO trace of the POG signal (vertical scale 0-I V/div, horizontal scale $5 \mathrm{~ms} /$ div) in $\mathrm{N}_{2}$ at a laser energy of $2 \mathrm{~mJ}$, gas pressure of 7 torr and discharge current of $12 \mathrm{~mA}$.

the excitation source. The discharge cell was made of glass having an inner diameter of $1 \mathrm{~cm}$ with tungsten electrodes at an inter-electrode separation of $\sim 1 \mathrm{~cm}$. Provision for continuous flow of the sample gas into the cell at rotary vacuum has also been provided. The schematic diagram of the experimental set up is shown in figure l(a). The discharge was produced by dc excitation in the cell with a ballast resistance in series across the tungsten electrodes. The discharge parameters were adjusted so as to get a steady discharge. The discharge was produced in gases like $\mathrm{N}_{2}, \mathrm{Ar}, \mathrm{NO}_{2}$ etc at various pressures and discharge currents. Pulsed dye laser beam was focussed on to the cathode using a lens. The POG signal taken out via a capacitor can be displayed on a CRO. A typical CRO trace of the POG signal obtained with $10 \mathrm{~ns}$ pulses at $564 \mathrm{~nm}$ radiation from the dye laser is shown in figure $1(\mathrm{~b})$. The signal amplitude was measured by varying laser energy, discharge current and gas pressure. 


\section{Results and discussion}

The variation of POG signal at $564 \mathrm{~nm}$ as a function of laser energy for different discharge currents at 5 torr and 7 torr of $\mathrm{N}_{2}$ gas pressures is studied. The work function of the electrode material (tungsten) is $4.3 \mathrm{eV}$ (Robert 1974) which approximately coincides with twice the photon energy at $564 \mathrm{~nm}$. Two-photon process has been confirmed from the log-log plot (iigure 2) of laser energy (I) versus signal amplitude $(\Delta V)$ which has a slope of $\sim 2$ implying that $\Delta V \sim I^{2}$. At low pressure, $(5$ torr) the slope is less than 2 indicating the presence of one-photon (OP) process along with two-photon (TP) process. The presence of one-photon process can be explained as due to the optogalvanic (OG) signal arising from the excitation of $\mathrm{N}_{2}$ molecules by the laser radiation at $564 \mathrm{~nm}$ wavelength. It should be noted that $564 \mathrm{~nm}$ emission coincides with 5,0 band of the first positive system (wavelength region $700-500 \mathrm{~nm}$ ) and 3,4 band of the Gaydon's Green system (wavelength region $640-500 \mathrm{~nm}$ ) in the spectrum of $\mathrm{N}_{2}$ molecules (Gaydon and Pearse 1965). The presence of OP and TP processes becomes more obvious when we make a least square fitting of the signal amplitude $\Delta V$ as a quadratic function of laser energy $I$ as,

$$
\Delta V=A I+B I^{2}
$$

Figure 3 shows the above curve fitted to experimental observations.

Variations of $A$ and $B$ coeflicients with discharge current for the two processes are shown in figure 4. At a pressure of 5 torr, the $A$-coefficient is negative at low currents. This behaviour is similar to $O G$ signal dependence on current where similar change of sign is observed (Van Veldhuizen et al 1984). As the discharge current increases, the $B$-coefficient decreases gradually showing that the OP process becomes more predominant at higher current (figure 4(a)). This fact is also revealed in the variation of slope in the log-log plot.

When the pressure is increased to 7 torr, the A-coefficient is always found to be negative, the magnitude of which increases with current (figure 4(b)). It has been shown that an increase in gas presssure leads to a more negative OG signal (Erez et al 1979). However the B-coeflicient is not so sensitive to current as in the case of discharge at 5 torr.
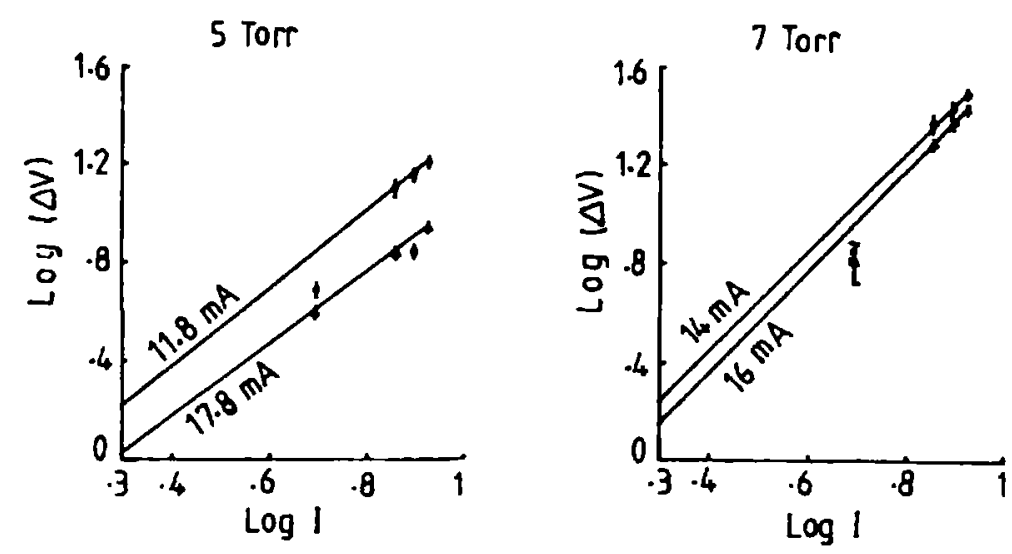

Figure 2 Log-log plot of laser energy vs POG signal amplitude at different discharge currents. 

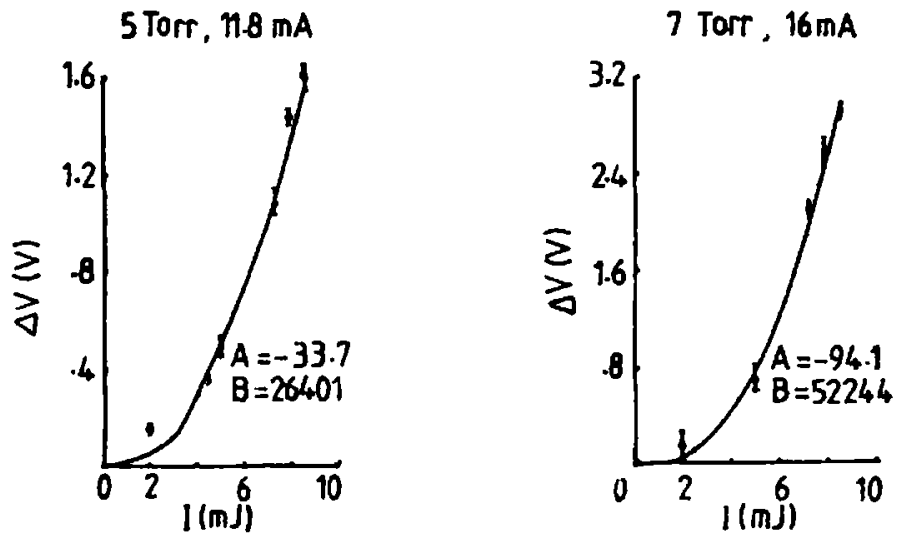

Figare 3. Curve for $\Delta V=A l+B I^{2}$ at 5 torr and 7 torr, $I$ is the laser energy per pulse. Points correspond to experimental observations.
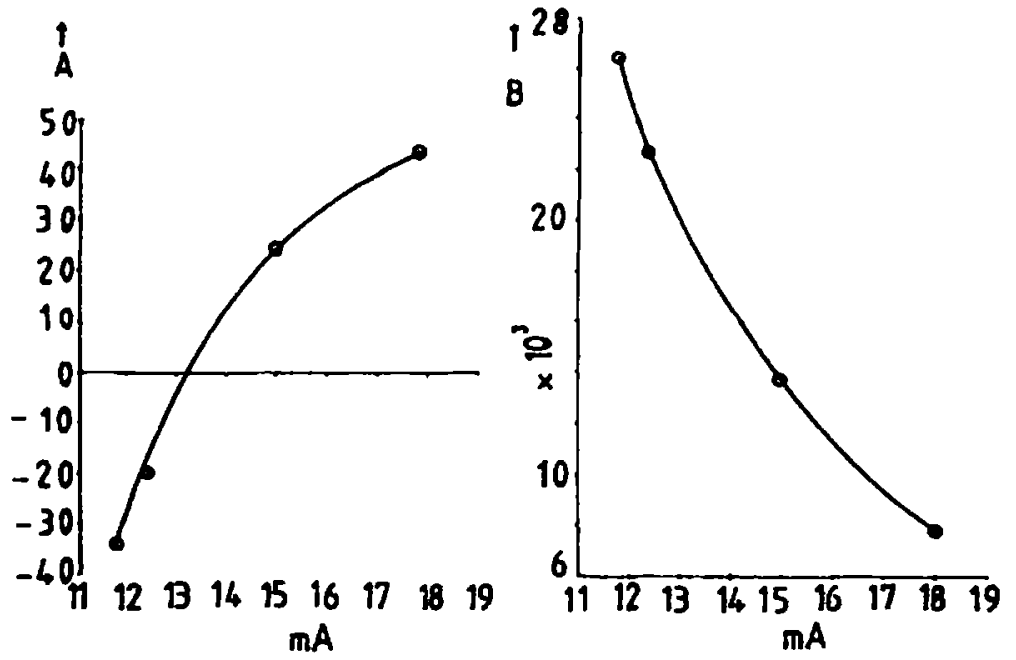

Figere $4(a)$. Variation of coeficients $A[$ in $V / J]$ and $B\left[\right.$ in $\left.V / J^{2}\right]$ with discharge current at 5 torr.
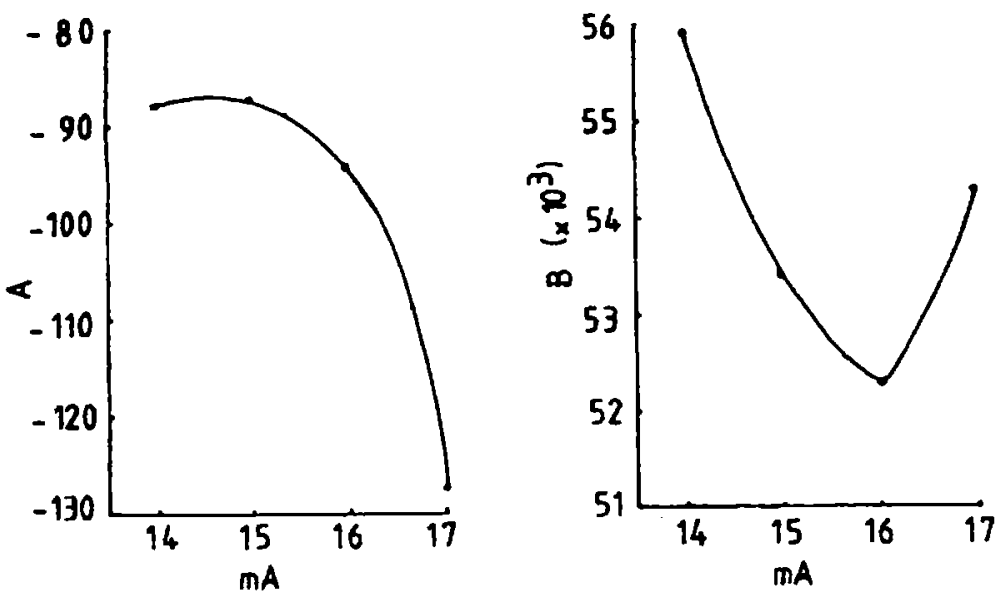

Figure $4(b)$ Variation of coeficients $A[$ in $V / J]$ and $B\left[\right.$ in $\left.V / J^{2}\right]$ with discharge current at 7 torr. 
In conclusion, we have observed two-photon induced photoemission optogalvanic effect using tungsten electrode in $\mathbf{N}_{2}$ gas discharge.

\section{Acknowledgements}

Authors are thankful to DST for financial assistance. Two of the authors (GP and AVRK) are grateful to CSIR for research fellowships.

\section{References}

Downey S W, Mitchell A and Gottscho R A 1988 J. Appl. Phys. 635280

Erez G, Lavi S and Miton E 1979 IEEE J. Quantwon Electron. 151328

Gaydon A G and Pearse R W B 1965 The identification of molecular spectra (London: Chapman \& Hall Ltd)

Green R B, Kellar R A, Luther G G, Schenck R K and Travis J J 1976 Appl. Phys. Lett. 29727

Robert C Weast 1974-1975 CRC Handbook of Chemistry and Physics 55 E-82

Smith K C and Schenck R K 1978 Chem. Phys. Lett. 55466

Van Veldhuizen E M, De Hoog F J and Schram D C 1984 J. Appl. Phys. 562047 